Article

\title{
Improved Genetic Algorithm Tuning Controller Design for Autonomous Hovercraft
}

\author{
Huu Khoa Tran ${ }^{1,2}$, Hoang Hai Son ${ }^{3}$, Phan Van Duc ${ }^{4}$, Tran Thanh Trang 5 \\ and Hoang-Nam Nguyen ${ }^{6, *(\mathbb{D})}$ \\ 1 Industry 4.0 Center, National Taiwan University of Science and Technology, Taipei 10607, Taiwan; \\ khoa.tran@mail.ntust.edu.tw \\ 2 Center for Cyber-Physical System Innovation, National Taiwan University of Science and Technology, \\ Taipei 10607, Taiwan \\ 3 Faculty of Mechanical, Electrical, Electronic and Automotive Engineering, Nguyen Tat Thanh University, \\ Ho Chi Minh City 700000, Vietnam; hhson@ntt.edu.vn \\ 4 Faculty of Automobile Technology, Van Lang University, Ho Chi Minh city 700000, Vietnam; \\ phanvanduc@vanlanguni.edu.vn \\ 5 Faculty of Engineering and Technology, Van Hien University, 665-667-669 Dien Bien Phu, \\ Ho Chi Minh city 700000, Vietnam; trangtt@vhu.edu.vn \\ 6 Modeling Evolutionary Algorithms Simulation and Artificial Intelligence, Faculty of Electrical \& Electronics \\ Engineering, Ton Duc Thang University, Ho Chi Minh City 700000, Vietnam \\ * Correspondence: nguyenhoangnam@tdtu.edu.vn
}

Received: 27 November 2019; Accepted: 26 December 2019; Published: 3 January 2020

\begin{abstract}
By mimicking the biological evolution process, genetic algorithm (GA) methodology has the advantages of creating and updating new elite parameters for optimization processes, especially in controller design technique. In this paper, a GA improvement that can speed up convergence and save operation time by neglecting chromosome decoding step is proposed to find the optimized fuzzy-proportional-integral-derivative (fuzzy-PID) control parameters. Due to minimizing tracking error of the controller design criterion, the fitness function integral of square error (ISE) was employed to utilize the advantages of the modified GA. The proposed method was then applied to a novel autonomous hovercraft motion model to display the superiority to the standard GA.
\end{abstract}

Keywords: modified GA; fuzzy-PID control; autonomous hovercraft; ISE criterion

\section{Introduction}

John Henry Holland, by imitating Darwin's biological evolution process, proposed the powerful stochastic global search method genetic algorithm (GA) first in 1975 [1,2]. Both the two reproduction mechanisms of genetic algorithm, including crossover and mutation, are used to find the convergence of optimal solutions. These values show an important effect on both behavior and performance. Therefore, GA instructions for choosing appropriate values are introduced by many researchers. In 1986, Grefenstette et al. proposed a method for optimizing the control gains for the genetic algorithm [3]. Then, in 1994, Srinivas and Patnaik demonstrated the adaptive probabilities of crossover and mutation [4]. In 1997, the bounded difficulty problems were considered by Harik [5]. Later in 2004, Zlochin et al. suggested model-based search for implementing combinatorial optimization [6]. In 2007, Zhang et al. adaptively adjusted crossover and mutation probabilities by utilizing a clustering-based technique [7]. Preceding this paper, GA and its innovations have been successfully deployed in a wide range of non-trivial complicated real-world issues, from optimization of flight control laws [8] to aerodynamic optimization problems [9]; from small wind turbine design [10] to path planning of a 
space-based of a manipulator system [11]; and from modeling collinear data [12] to ship navigation in collision situations [13].

The genetic algorithm optimization offers a powerful methodology for solving single to multivariable problems. Each GA represents a problem solution encoded by the form of binary strings or chromosomes. Its fitness is employed to measure how good of one solution by increasing the best bit patterns. Hence, the maxima/minima of fitness function value are then optimized during the GA course.

Nevertheless, the standard GA (denoted shortly as sGA) still has some drawbacks; for example, low convergence speed and premature convergence because of requiring hundreds of updated generations. Hence, we proposed a modified genetic algorithm (denoted shortly as mGA) to improve the optimal process. This modified algorithm, just in short operating generations [1,2,14], can improve the global search efficiency and increase the convergence speed of the optimal control design. Next, the proposed control design was a fuzzy-proportional-integral-derivative (F-PID) control [15-25] that comprises the fuzzy logic control (FLC) and the common PID controller. The first, fuzzy term is employed to increase the stability and the robustness of the controller design by tuning the membership functions and by selecting suitable methods of fuzzification and defuzzification [15-19,24,25]. The second, PID control term is separated in two small sub-terms: the PD is employed to maintain the system stability while the term I is utilized to eliminating the steady-state error of the controlled system response [20-23]. Based on the calculation of criterion error of the control system, the "integral of square error" (ISE) [20-22] was chosen as a fitness function to show the controller performance index.

A hovercraft, also known as an air cushion vehicle (ACV), moves smoothly on any surface [26-31] from the ground-land to the mud, water, sand, and even on ice. Because a hovercraft is very active and agile, its models was chosen for the control implementation.

To summarize, the modified GA is proposed to take advantages of the fuzzy-PID controller design in shorter operation period. Thus, the dynamic of Hovercraft models could be mobility in high stability with fast response and less error.

\section{Hovercraft Prototype Model}

A hovercraft, which is known as an underactuated system and named an air cushion vehicle $(\mathrm{ACV})$, has rotors and a cushion, where inside air pressure enables floating and smooth movement on any surface [26-31], such as land, mud, water, sand, and even on ice. The hovercraft is very active and agile; hence, it is applied widely in the coastguard, army, rescue operations, civil engineering, etc. The hovercraft is mounted with a single tilt servomotor on the fin tail. As shown in Figure 1, the rotor duct fan of the hovercraft is settled along the y-axis, while the propeller is attached along the z-axis. Firstly, the lift propeller provides the internal cushion pressure to lift up in a long operation period. Next, the forward moving is created by the rear rotor duct-fan. Finally, the turning typically is operated by directing the thrust airflow through rotor duct fan, which is steered by a tilt servomotor placed at the rear. The subsequently generated momentum is used to maneuver the craft. Although many modern technologies are utilized, the hovercraft still requires an advanced maneuvering system to achieve optimized performance.

The hovercraft dynamic models were derived in [28-31] using right-hand convention coordinate systems. The positive $x$-axis covers the lateral factors, namely, sway motion or surge position, while the $y$-axis is the direction along the hovercraft body, covering surge motion or sway position; and the positive z-axis defines the downwards direction. The hovercraft's kinematics can be expressed as Equation (1):

$$
\left\{\begin{array}{c}
\dot{x}=u \cos \psi-v \sin \psi \\
\dot{y}=v \cos \psi+u \sin \psi \\
\dot{\psi}=r
\end{array}\right.
$$

where $u \in R$ and $v \in R$ represent linear velocities in surge direction and sway direction, respectively. The angular velocity is represented as $r \in R$. Using Equation (1), we can derive the kinetic energy $T$ 
and potential energy $V$ to define the Lagrange $L=T-V$ by applying Euler-Lagrange formulation as in following equation:

$$
M(q) \dot{q}+C(q, \dot{q}) q=\left[\begin{array}{l}
F \\
\tau \\
0
\end{array}\right]
$$

where $\tau$ is the torque in yaw rotation and $F$ is the force acting along surge direction.

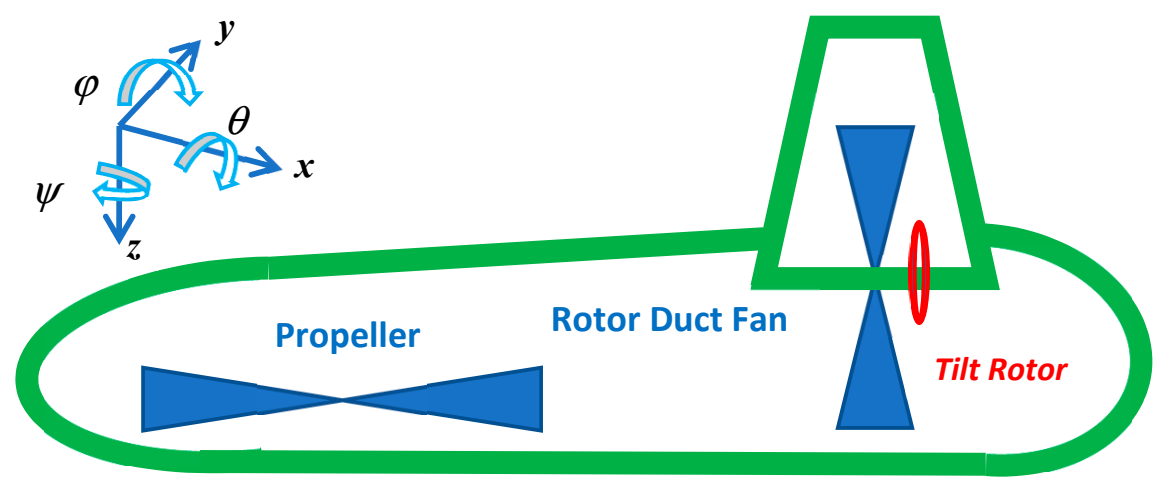

Figure 1. The hovercraft configuration model.

\section{Modified GA Optimal Controller Gains}

\subsection{The Controller Design}

Fuzzy logic concepts are extremely modest but powerful and effective for applications in the control of various machines. The fuzzy control takes advantages in stability and robustness since its aptitude is deal with the nonlinear and uncertain systems. Fuzzy control rules are designed based on the center of area method (COA) for defuzzification and Mandani's MIN-MAX inference engine type. It includes the tracking error $e(t)$ and the differential tracking error $d e(t)$ as the inputs. The fuzzy control output to PID control is defined as $e_{F u z z y}(t)$. Seven partitions of the fuzzy rule-table control, which exploit the triangular membership functions (as shown in Figure 2), are noted in Table 1, which include negative small (NS), negative medium (NM), negative big (NB), positive big (PB), positive medium (PM), positive small (PS), and zero (Z).

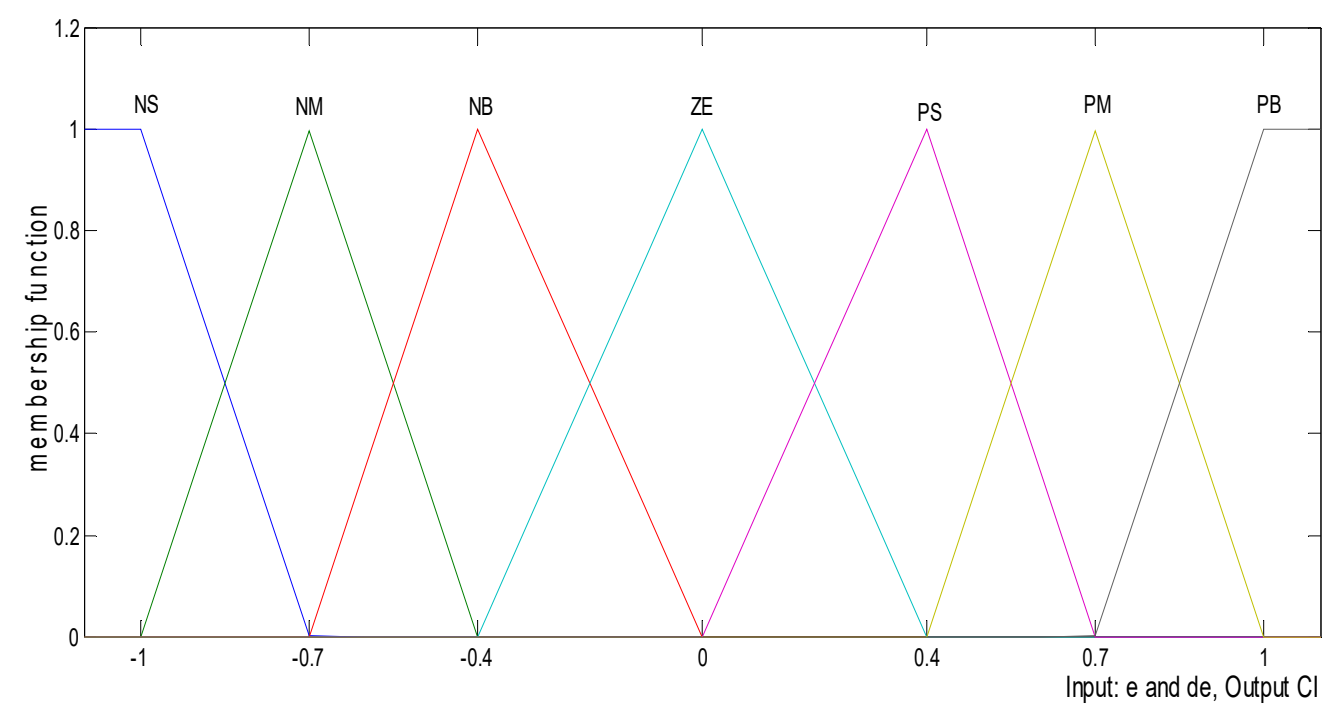

Figure 2. The fuzzification membership function. 
Table 1. Fuzzy rule-table.

\begin{tabular}{|c|c|c|c|c|c|c|c|c|}
\hline \multirow{2}{*}{\multicolumn{2}{|c|}{$e_{F u z z y}(t)$}} & \multicolumn{7}{|c|}{$e(t)$} \\
\hline & & NB & NM & NS & $\mathbf{Z}$ & PS & $\mathbf{P M}$ & PB \\
\hline \multirow{7}{*}{$d e(t)$} & PB & Z & PS & PM & PB & PB & PB & PB \\
\hline & PM & NS & $\mathrm{Z}$ & PS & PM & PB & PB & PB \\
\hline & PS & NM & NS & Z & PS & PM & PB & PB \\
\hline & Z & NB & NM & NS & Z & PS & PM & PB \\
\hline & NS & NB & NB & NM & NS & Z & PS & PM \\
\hline & NM & NB & NB & NB & NM & NS & $\mathrm{Z}$ & PS \\
\hline & NB & NB & NB & NB & NB & NM & NS & $\mathrm{Z}$ \\
\hline
\end{tabular}

PID controllers are designed to satisfy dynamic response, and reduce and/or eliminate error of physical empirical systems. Thus, the fuzzy-PID controller $[24,25]$ output $u$ to the system is proposed and expressed on Equation (3) as:

$$
u=u_{F u z z y}+u_{P I D}=u_{F u z z y}+k_{p} \times e+k_{I} \int e d t+k_{D} \times \frac{d}{d t} e(t)
$$

where $u_{F u z z y}$ and $u_{P I D}$ are the Fuzzy and PID controller output signals. $k_{P}, k_{I}, k_{D}$ are the PID controller proportional, integral and derivative constants.

The fuzzy-PID controls is highly effective, as it is simple, has less overshoot, and is able to eliminate the steady state error, and especially smooth control signal. Its block diagram is demonstrated in Figure 3.

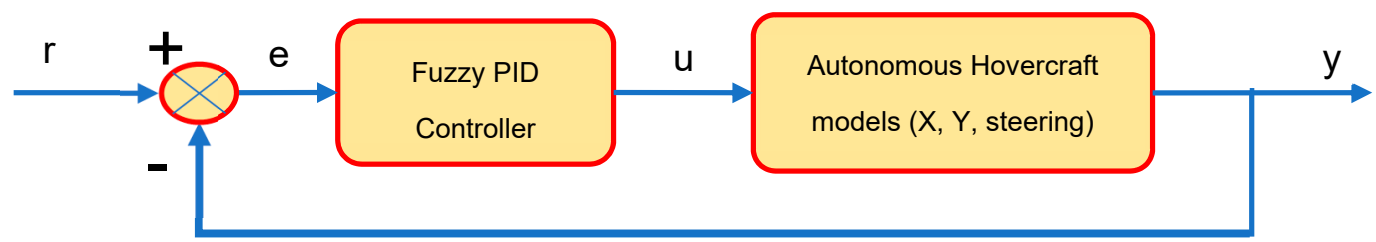

Figure 3. The controller block diagram.

\subsection{Improved GA in the Optimization Process}

The standard GA's characteristics [3], which includes three main parameters-mutation rate $(R m)$, crossover rate $(R c)$, and population size $(N)$-were employed to verify the optimized gains of the proposed control system. This optimal program usually takes hundreds of generations to update and find out convergence gains. Therefore, the GA is powerful, but it still has a critical drawback, which has been investigated for a solution by many researchers. By checking the GA process, it was found out that chromosome decode is not compulsory due to the evaluation of the cost function $[1,2,14]$. Hence, we proposed the novel modified GA method where the chromosome decoding step is totally neglected, as shown in Figure 4. This improvement makes modified GA more effective than the conventional GA in the aspects of storage (less) and convergence speed (naturally higher) [1,2,14]. The optimal process of the modified GA to Fuzzy-PID control parameters using the ISE (integral of square errror) fitness function is formulated as ISE $=\int_{0}^{\infty} e^{2}(t) d t$. 


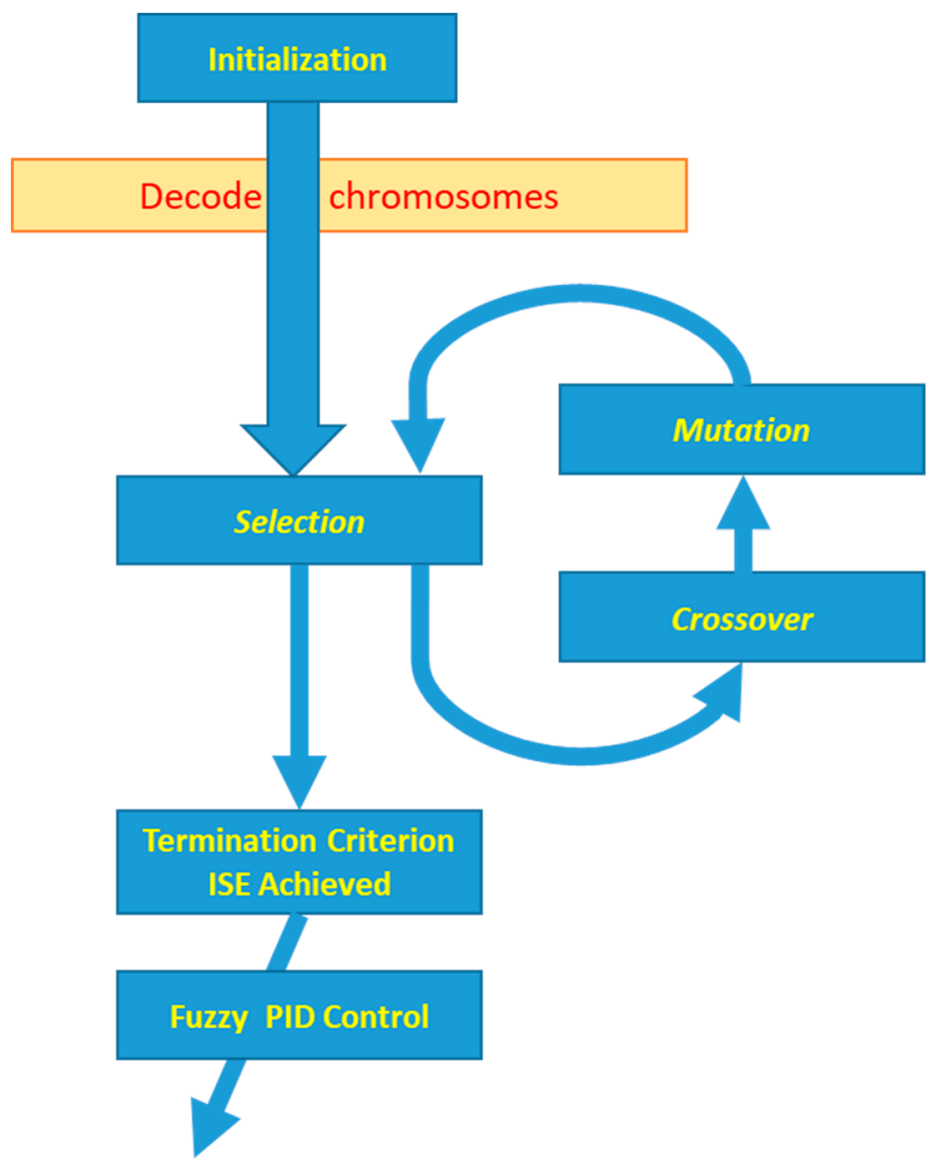

Figure 4. The proposed fuzzy-PID-mGA (fuzzy-proportional-integral-derivative modified genetic algorithm; the decode chromosome step is neglected).

\section{Numerical Simulation Results}

The autonomous hovercraft simulation parameters were derived from [28-31] and denoted with the mass $m=2.1 \mathrm{~kg}$ and the inertia moment $I=0.000257 \mathrm{~kg} . \mathrm{m}^{2}$. The modified GA had the mutation rate $(R m)$ of 0.08 , the crossover rate $(R c)$ of 0.95 , and one hundred individuals $(n=100)$. We proposed to tune the fuzzy-PID controller gains in only 20 population-generations of the simulation process. The PID gains were chosen arbitrary initialized in range $(0,50)$ while $e$ and de of fuzzy control changed slightly around 1 ; the control parameters after optimization by the mGA are in Table 2. In comparison with the standard GA, the modified GA operated in shorter generations (just 20 generations) and rapidly updated the convergence speed of fitness function.

The hovercraft was tested by moving forward (x-direction); stability was tested when it was attacked on the side, as it would be by a wave or the wind (y-direction); and steering was by pilot control (z-direction). Hence, the subsystems of the hovercraft are separated in each channel for the strategy of design the controller. Performance of the proposed controller of the autonomous Hovercraft motions are shown on surge position on x-axis in Figure 5, sway position on y-axis in Figure 6, and yaw angle on z-axis in Figure 7, respectively. The numerical simulation result, achieved in just after 20 generations, clearly proves that the proposed methodology gives a fast response, less error, and zero overshoot. Moreover, the ISE fitness function, as shown in part $b$ of each of Figures 5-7, gives the better process of the minimum error. In all three of Figures $5-7$, the modified GA error from start to finish is faster than the standard GA, as shown on Table 3. The 1/Fitness error being larger means that the speed of the convergence from start toward finish is faster. All the simulation performance work was done on the MATLAB/Simulink platform (R2018a, MathWorks, Natick, MA, USA). 


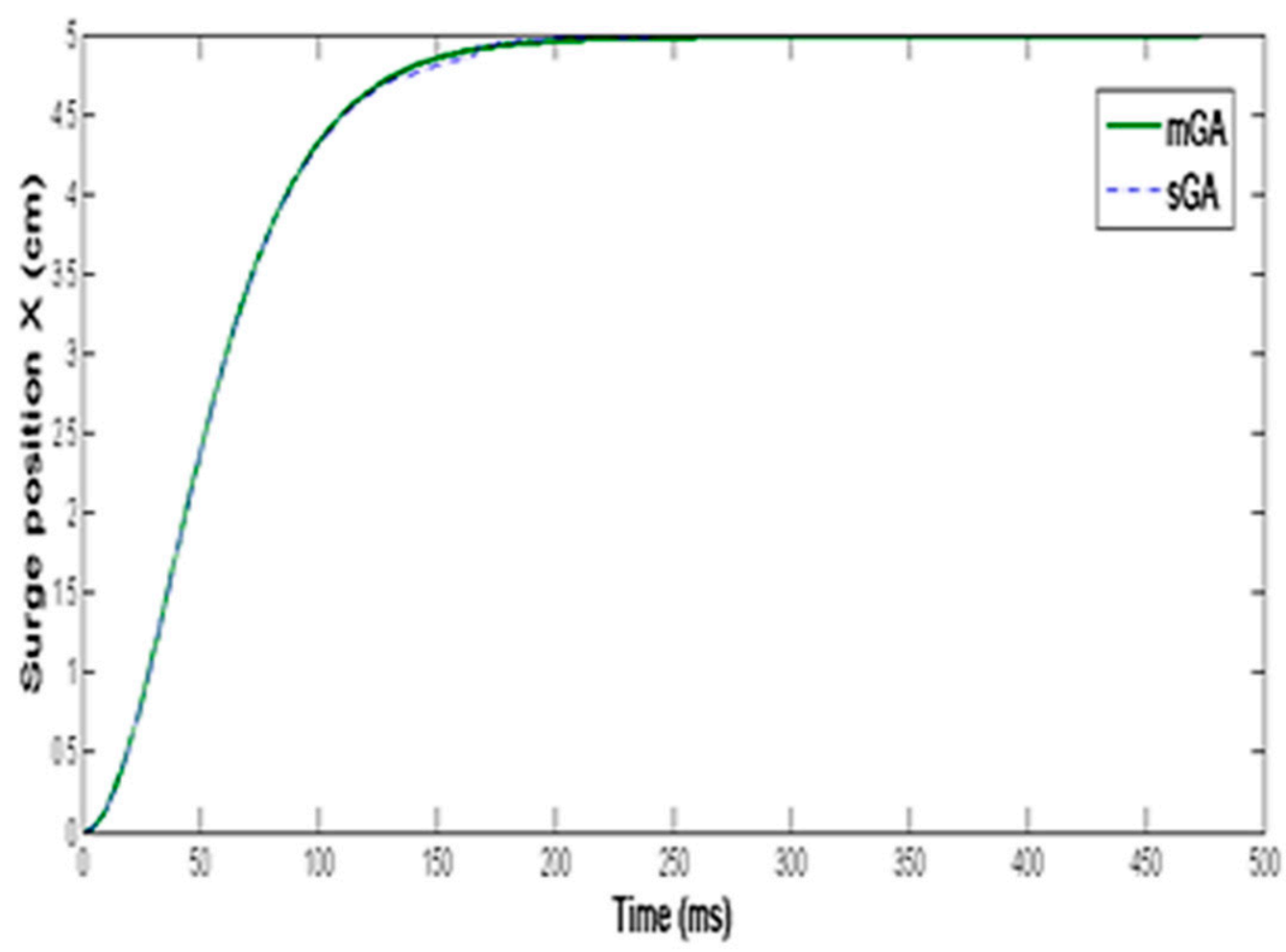

(top)

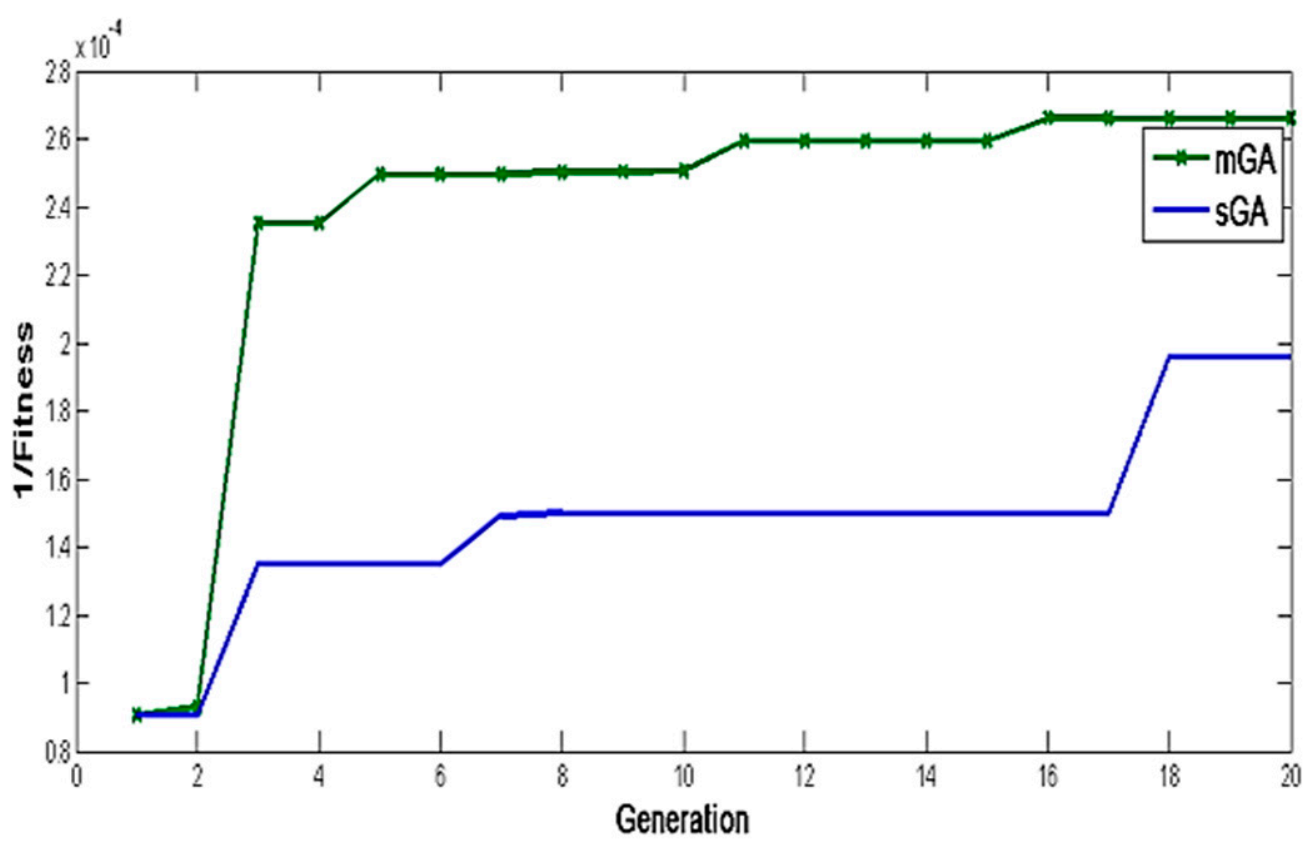

(bottom)

Figure 5. (Top) Surge-position control; (bottom) improved GA (mGA) versus standard GA (sGA) fitness functions. 


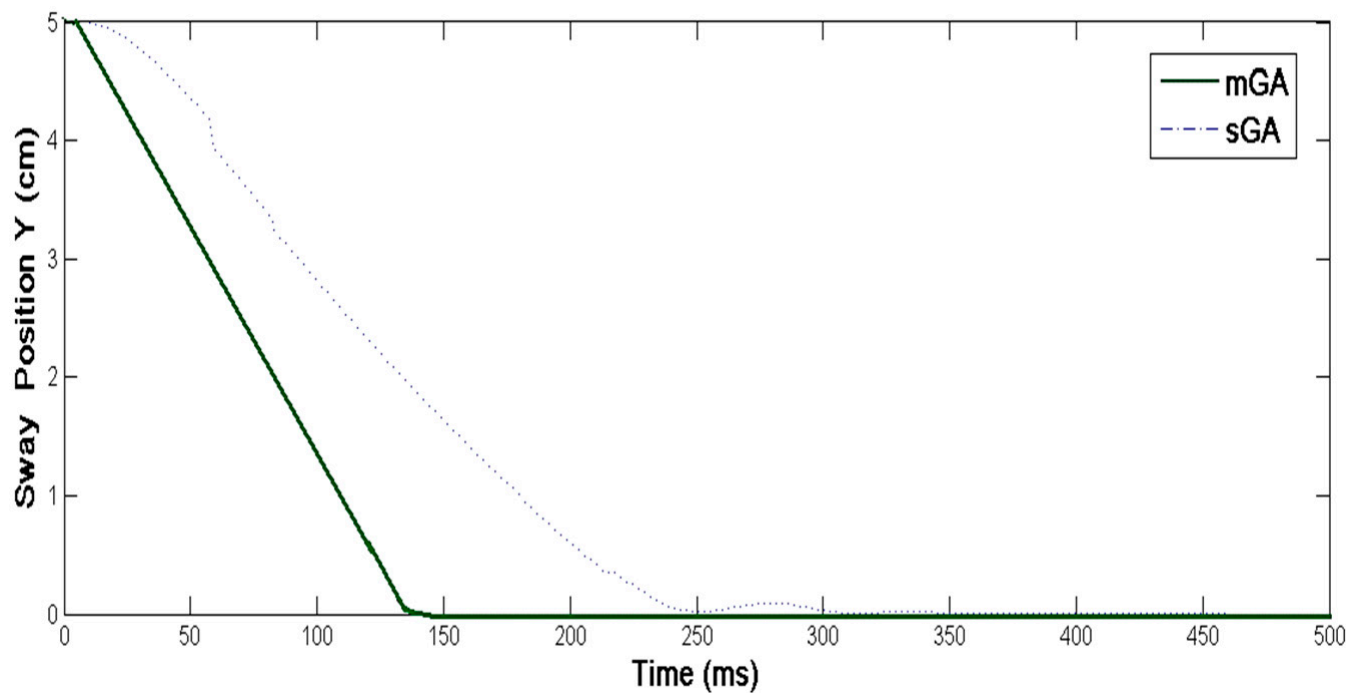

(top)

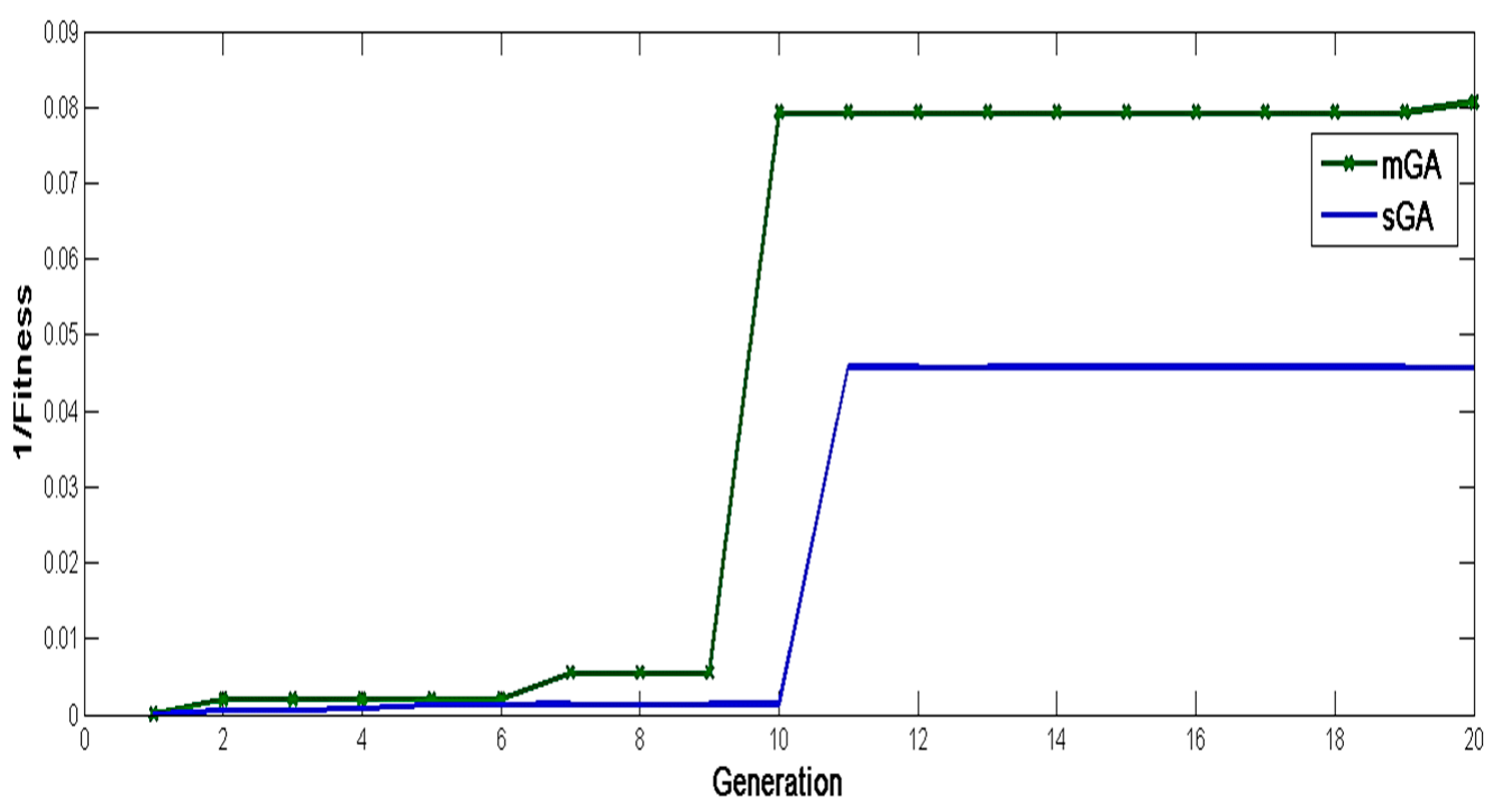

(bottom)

Figure 6. (Top) Sway-position control; (bottom) improved GA (mGA) versus standard GA (sGA) fitness functions.

Table 2. The fuzzy-PID-mGA tuning results.

\begin{tabular}{ccccccc}
\hline & Generation & $\boldsymbol{e}$ & $\boldsymbol{d e}$ & $\boldsymbol{K}_{\boldsymbol{I}}$ & $\boldsymbol{K}_{\boldsymbol{P}}$ & $\boldsymbol{K}_{\boldsymbol{D}}$ \\
\hline Surge position x $(5 \mathrm{~cm})$ & 20 & 1 & 1 & 41.61 & 8.75 & 1.42 \\
Sway position y $(5 \mathrm{~cm})$ & 20 & 0.98 & 1.05 & 29.3 & 3.32 & 0.66 \\
Steering-Yaw angle $(5$ degree $)$ & 20 & 1.1 & 1.03 & 32.8 & 5.91 & 3.26 \\
\hline
\end{tabular}

Table 3. The error fitness function by generations.

\begin{tabular}{lcccccc}
\hline \multicolumn{1}{c}{ 1/Fitness Error } & i Gene-Ration & sGA & mGA & i Gene-Ration & sGA & mGA \\
\hline Surge position $x(5 \mathrm{~cm})$ & 3 & 0.00137 & 0.00236 & 20 & 0.0018 & 0.00263 \\
Sway position y $(5 \mathrm{~cm})$ & 10 & 0.045 & 0.08 & 20 & 0.045 & 0.081 \\
Steering-Yaw angle (5 degree) & 10 & 0.00035 & 0.00067 & 20 & 0.00036 & 0.00095 \\
\hline
\end{tabular}




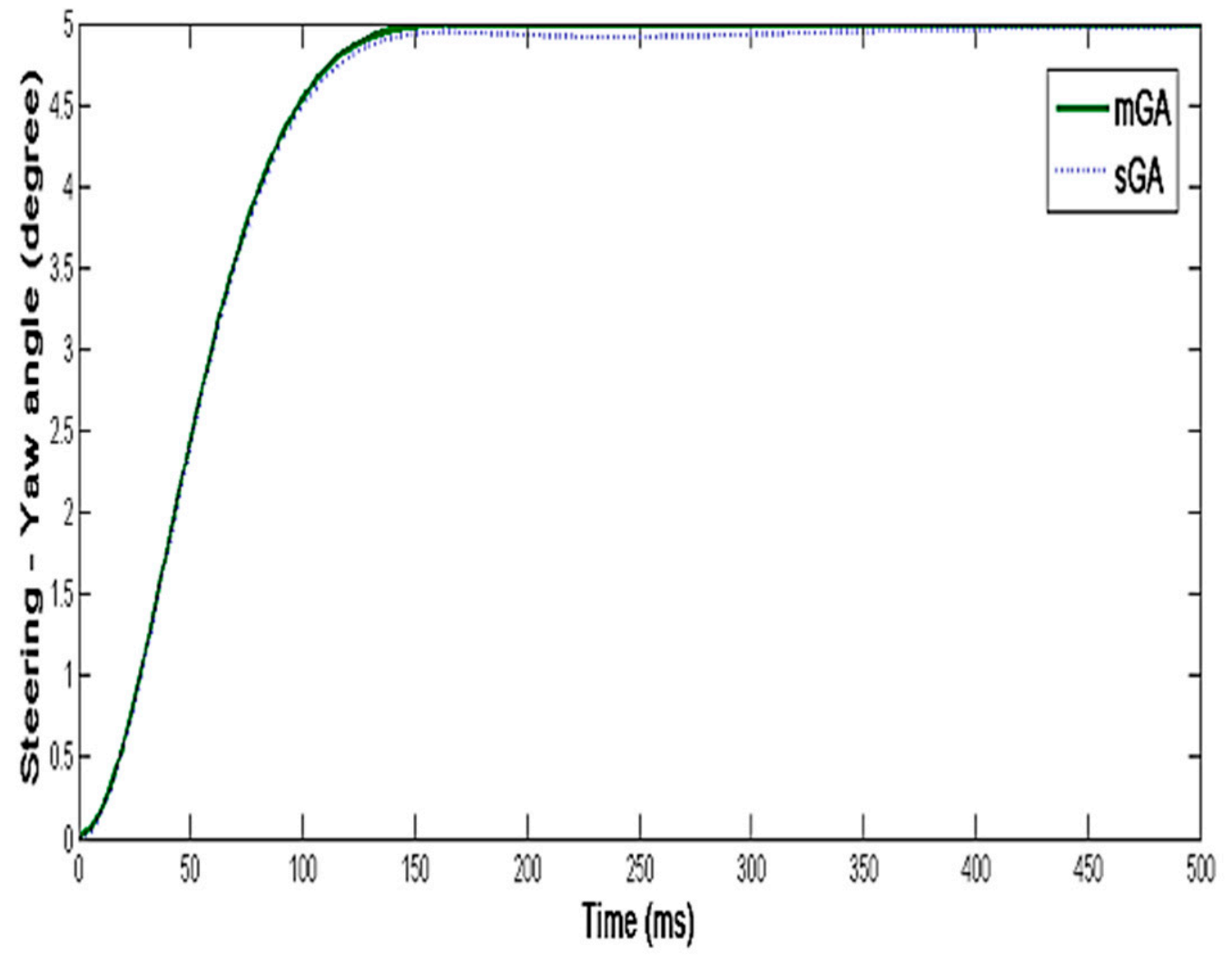

(top)

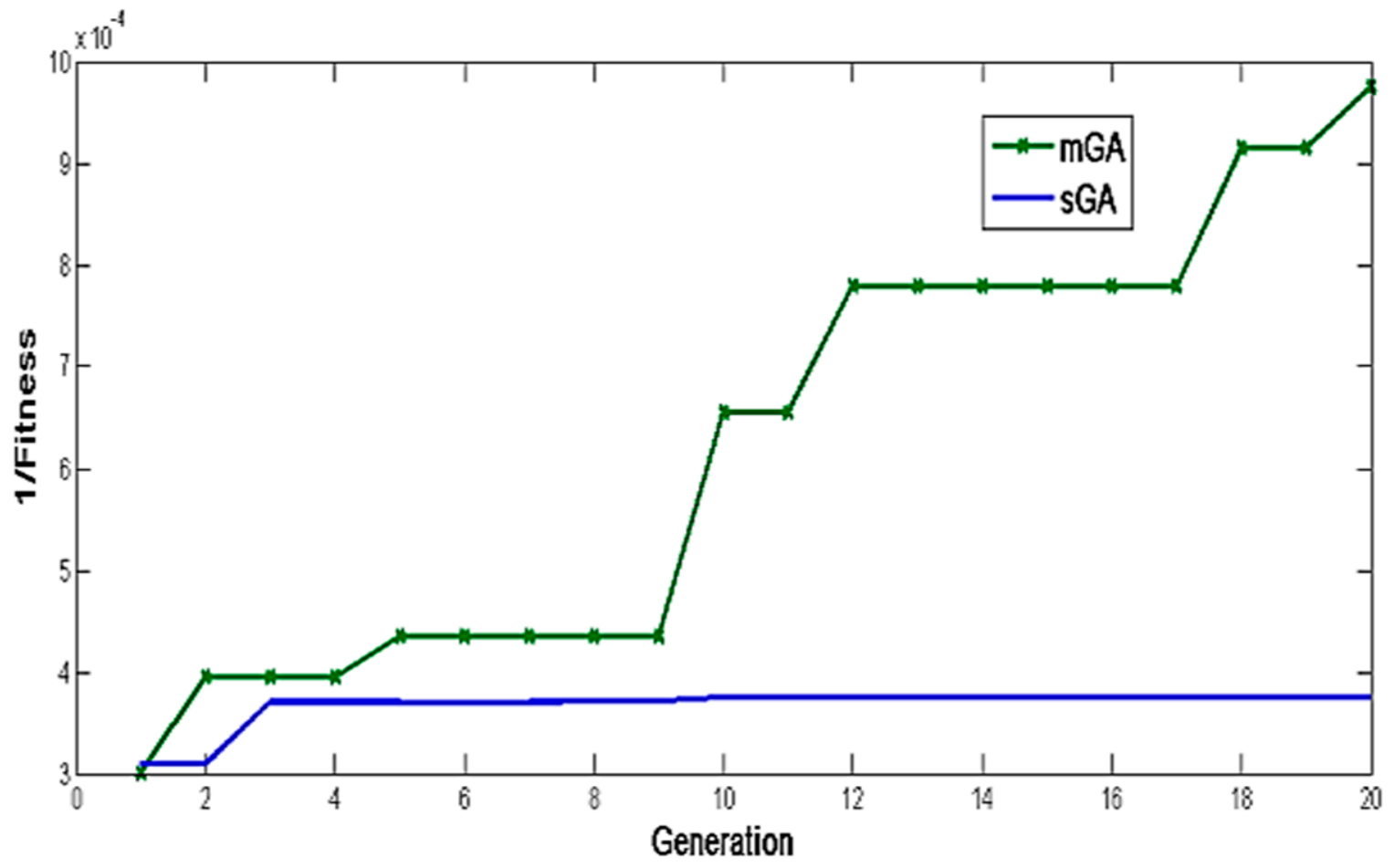

(bottom)

Figure 7. (Top) Steering-Yaw control; (bottom) improved GA (mGA) versus standard GA (sGA) fitness functions. 


\section{Conclusions}

In this study, genetic algorithms, during a short operation period, were utilized to optimize the parameters of an autonomous hovercraft controller. The proposed method achieved good performances in terms of response (fast), stability (high), error (low), and overshoot (none at all). In addition, the improved GA methodology, which was implemented by make some simple changes inside the standard GA's process that neglects/eliminates the chromosome decode step, displayed better performances in convergence speed. Especially, the modified GA can update the error fitness function in a smaller number of generations. It is undeniable that the improved GA is valuable in the optimization processes, particularly in optimizing the controller parameters. In further research, the authors would like to enhance the efficiency of the modified GA by using another error criteria, such as: ITSE (integral of time weighted square error) and MSE (mean square error). The disturbances attack to system models will be also considered to verify the efficiency of the process of optimizing control parameters.

Author Contributions: Methodology, H.K.T.; Supervision, H.K.T.; Visualization, H.K.T.; Resources, P.V.D., H.H.S.; Validation, T.T.T.; Writing-Review \& Editing, H.-N.N. All authors have read and agreed to the published version of the manuscript.

Funding: This work was financially supported by the "Center for Cyber-physical System Innovation" from The Featured Areas Research Center Program within the framework of the Higher Education Sprout Project by the Ministry of Education (MOE) in Taiwan.

Conflicts of Interest: The authors declare that there is no conflict of interests regarding the publication of this paper.

\section{References}

1. Yang, X.-S. Engineering Optimization: An Introduction with Metaheuristic Applications; John Wiley \& Sons, Inc.: Hoboken, NJ, USA, 2010.

2. Haupt, R.L.; Haupt, S.E. Practical Genetic Algorithms, 2nd ed.; John Wiley \& Sons, Inc.: Hoboken, NJ, USA, 2004.

3. Grefenstette, J.J. Optimization of Control Parameters for Genetic Algorithms. IEEE Trans. Syst. Man Cybern. 1986, 16, 122-128. [CrossRef]

4. Srinivas, M.; Patnaik, L. Adaptive Probabilities of Crossover and Mutation in Genetic Algorithms. IEEE Trans. Syst. Man Cybern. 1994, 24, 656-667. [CrossRef]

5. Harik, G. Learning Linkage to Efficiently Solve Problems of Bounded Difficulty Using Genetic Algorithms. Ph.D. Thesis, Dept. Computer Science, University of Michigan, Ann Arbor, MI, USA, 1997.

6. Zlochin, M.; Birattari, M.; Meuleau, N.; Dorigo, M. Model-Based Search for Combinatorial Optimization: A Critical Survey. Ann. Oper. Res. 2004, 131, 373-395. [CrossRef]

7. Zhang, J.; Chung, H.; Lo, W.L. Clustering-Based Adaptive Crossover and Mutation Probabilities for Genetic Algorithms. IEEE Trans. Evol. Comput. 2007, 11, 326-335. [CrossRef]

8. Al-Asasfeh, A.; Hamdan, N.; Abo-Hammour, Z. Flight Control Laws Verification Using Continuous Genetic Algorithms. ISRN Robot. 2013, 2013. [CrossRef]

9. Antunes, A.P.; Azevedo, J.L.F. Studies in Aerodynamic Optimization Based on Genetic Algorithms. J. Aircr. 2014, 51, 1002-1012. [CrossRef]

10. Hamdan, M.; Abderrazzaq, M.H. Optimization of Small Wind Turbines Using Genetic Algorithms. Int. J. Appl. Metaheuristic Comput. 2016, 7, 50-65. [CrossRef]

11. Chen, Z.; Zhou, W. Path Planning for a Space-Based Manipulator System Based on Quantum Genetic Algorithm. J. Robot. 2017. [CrossRef]

12. Tang, J.; Zhang, J.; Wu, Z.; Liu, Z.; Chai, T.; Yu, W. Modeling Collinear Data Using Double-Layer GA-Based Selective Ensemble Kernel Partial Least Squares Algorithm. Neurocomputing 2017, 219, 248-262. [CrossRef]

13. Ni, S.; Liu, Z.; Cai, Y. Ship Manoeuvrability-Based Simulation for Ship Navigation in Collision Situations. J. Mar. Sci. Eng. 2019, 7, 90. [CrossRef]

14. Tran, H.K. Modified GA Tuning IPD Control for a Single Tilt Tri-Rotors UAV. Int. Rev. Aerosp. Eng. (IREASE) 2018, 11, 1-5. [CrossRef]

15. Passino, K.M.; Yurkovich, S. Fuzzy Control; Addison-Wesley Reading: Boston, MA, USA, 1998.

16. Precup, R.-E.; Preitl, S. PI-Fuzzy Controllers for Integral Plants to Ensure Robust Stability. Inf. Sci. 2007, 177, 4410-4429. [CrossRef] 
17. Sanchez, E.N.; Becerra, H.M.; Velez, C.M. Combining Fuzzy, PID and Regulation Control for an Autonomous Mini-Helicopter. Inf. Sci. 2007, 177, 1999-2022. [CrossRef]

18. Feng, J.Z.; Li, J.; Yu, F. GA-Based PID and Fuzzy Logic Control for Active Vehicle Suspension System. Int. J. Automot. Technol. 2003, 4, 181-191.

19. Juang, Y.T.; Chang, Y.T.; Huang, C.P. Design of Fuzzy PID Controllers Using Modified Triangular Membership Functions. Inf. Sci. 2008, 178, 1325-1333. [CrossRef]

20. Martins, F.G. Tuning PID Controllers Using the ITAE Criterion. Int. J. Eng. Educ. 2005, 21, 867-873.

21. Skogestad, S. Simple Analytic Rules for Model Reduction and PID Controller Tuning. J. Process. Control. 2003, 13, 291-309. [CrossRef]

22. Tan, W.; Liu, J.; Chen, T.; Marquez, H.J. Comparison of Some Well-Known PID Tuning Formulas. Comput. Chem. Eng. 2006, 30, 1416-1423. [CrossRef]

23. Wu, Y.; Zhao, X.; Li, K.; Zheng, M.; Li, S. Energy Saving-Another Perspective for Parameter Optimization of $\mathrm{P}$ and PI Controllers. Neurocomputing 2016, 174, 500-513. [CrossRef]

24. Tóthová, M.; Pitel', J. Simulation of Fuzzy Adaptive Position Controllers for Pneumatic Muscle Actuator. In Proceedings of the IEEE 13th International Symposium on Intelligent Systems and Informatics (SISY), Subotica, Serbia, 17-19 September 2015; pp. 55-59.

25. Phu, D.X.; Choi, S.-B. A New Adaptive Fuzzy PID Controller Based on Riccati-Like Equation with Application to Vibration Control of Vehicle Seat Suspension. Appl. Sci. 2019, 9, 4540. [CrossRef]

26. Sira-Ramirez, H.; Ibanez, C.A. On the Control of the Hovercraft. Dyn. Control. 2000, 10, 151-163. [CrossRef]

27. Balemi, S.; Bucher, R.; Guggiari, P.; Furlan, I.; Kottmann, M.; Chapuis, J. Rapid Control of Prototyping of a Hovercraft. In Proceedings of the MSy'02, Embedded Systems Conference, Shanghai, China, 11-16 October 2002.

28. Rashid, M.Z.A.; Aras, M.S.M.; Kassim, M.A.; Ibrahim, Z.; Jamali, A. Dynamic Mathematical Modeling and Simulation Study of Small Scale Autonomous Hovercraft. Int. J. Adv. Sci. Technol. 2012, 46, 95-114.

29. Chaos, D.; Moreno-Salinas, D.; Muñoz-Mansilla, R.; Aranda, J. Nonlinear Control for Trajectory Tracking of a Nonholonomic RC-Hovercraft with Discrete Inputs. Math. Probl. Eng. 2013, 2013, 589267. [CrossRef]

30. Garcia, D.I.; White, W.N. Control Design of an Unmanned Hovercraft for Agricultural Applications. Int. J. Agric. Biol. Eng. 2015, 8, 72-79.

31. Cabecinhas, D.; Batista, P.; Olivera, P.; Silvestre, C. Hovercraft Control with Dynamic Parameters Identification. IEEE Trans. Control. Syst. Technol. 2018, 26, 785-796. [CrossRef] 